\title{
Study on workloads of human care worker with the introduction of IT system - the characteristics of work loads by observational research and the suggestions for KAIZEN
}

\author{
Yuki MIZUNO a,b, ${ }^{\text {, }}$, Toru YOSHIKAWA ${ }^{\mathrm{b}}$, Fumiko MATSUDA ${ }^{\mathrm{b}}$, Yuriko TAKEUCHI $^{\mathrm{b}}$, Nobuyuki \\ MOTEGI $^{\mathrm{b}}$, Thor IKEGAMI ${ }^{\mathrm{c}}$, Kazuhiro SAKAI ${ }^{\mathrm{b}}$ \\ a Toyo Gakuen University Center for Liberal Arts, Tokyo, Japan \\ ${ }^{\mathrm{b}}$ Department of Research, The Institute for Science of Labour, Kawasaki, Japan \\ c ITI Consultancy, Koshigaya, Japan
}

\begin{abstract}
The purpose of this study was to clarify the characteristic of workloads on human care worker with the introduction of IT system, and suggested the support measures for KAIZEN in Japan. The investigation method is workflow line and hearing with a focus on work observation. The objects were 8 human care workers of the acute hospital that introduced an electronic system. By the introduction of the electronic chart, the nurse station sojourn time decreased, sickroom sojourn time increased, and time about direct nursing care to a patient increased. In addition, access to patient information became easy, and the offer of the health care service based on correct information came to be possible in real time. By The point of workflow line, it was effect that moving lengths decreased in order to install the electronic chart in patients' rooms. Though, it was a problem that it hasn't formed where to place the instruments such as sphygmomanometer, clinical thermometer and others.
\end{abstract}

Keywords: work loads, KAIZEN, human care worker, workflow line, IT system

\section{Introduction}

In recent years, the medical and welfare institutions introducing the IT systems such as electronic charts increase. It is reported that improve such as efficiency of record and intelligence, increase of the nursing care time and increase of the conference by the introduction of the electronic chart. Therefore, this study investigated the characteristic of workloads on human care worker with the introduction of IT system, and suggested the support measures for KAIZEN.

\section{Method}

\subsection{Investigation method}

The investigation method is workflow line and hearing with a focus on work observation.

\subsection{Objects}

The objects were 8 human care workers (workers over 3 years experience were 4 , and new faces less

\footnotetext{
*Corresponding author. E-mail: XLH02731@nifty.ne.jp
} 
than 2 years were 4 ) of the acute hospital that introduced an electronic system into a few years ago.

\section{Results}

As a result of observation investigation, in the institution, the paper charts and the paper slip were mixed after introduction an electronic system.

The electronic chart was installed in not only the nurse station but also the patients' rooms and was able to perform the entry or the intelligence of the medical record in front of a charge patient. In other words, the introduction of IT system became able to cope with the change of the condition of the patient immediately.

By the introduction of the electronic chart, the nurse station sojourn time decreased, sickroom sojourn time increased, and time about direct nursing care to a patient increased. In addition, access to patient information became easy, and the offer of the health care service based on correct information came to be possible in real time. Furthermore, by promotion of sharing information, much information of patient provided. It showed the difference between using IT system of skilled worker and non-skilled worker (Fig.1). The nurse with more than three years of experience recorded and undertaken information gathering diligently and information management was completed within working hours. The nurse within two years went into overtime because they intensively used IT medical record after the end of working hours. By the years of experience, overtime hours increase, and it is a problem of health or fatigue.

\section{Consideration}

As for the cognitive loads, the work included rearranging and confirmation of the enormous information with the IT system, it was necessary to skill how to make nursing-care plan (work schedule) of the day. The physical loads were being over hours work and being lock of rest time. By The point of workflow line, it was effect that moving lengths decreased in order to install the electronic chart in patients' rooms. Though 、 it was a problem that it hasn't formed where to place the instruments such as sphygmomanometer, clinical thermometer and others. As for the emotional loads, in case of inexperienced nurses, there was much useless movement and was not almost medical record management during duty. 


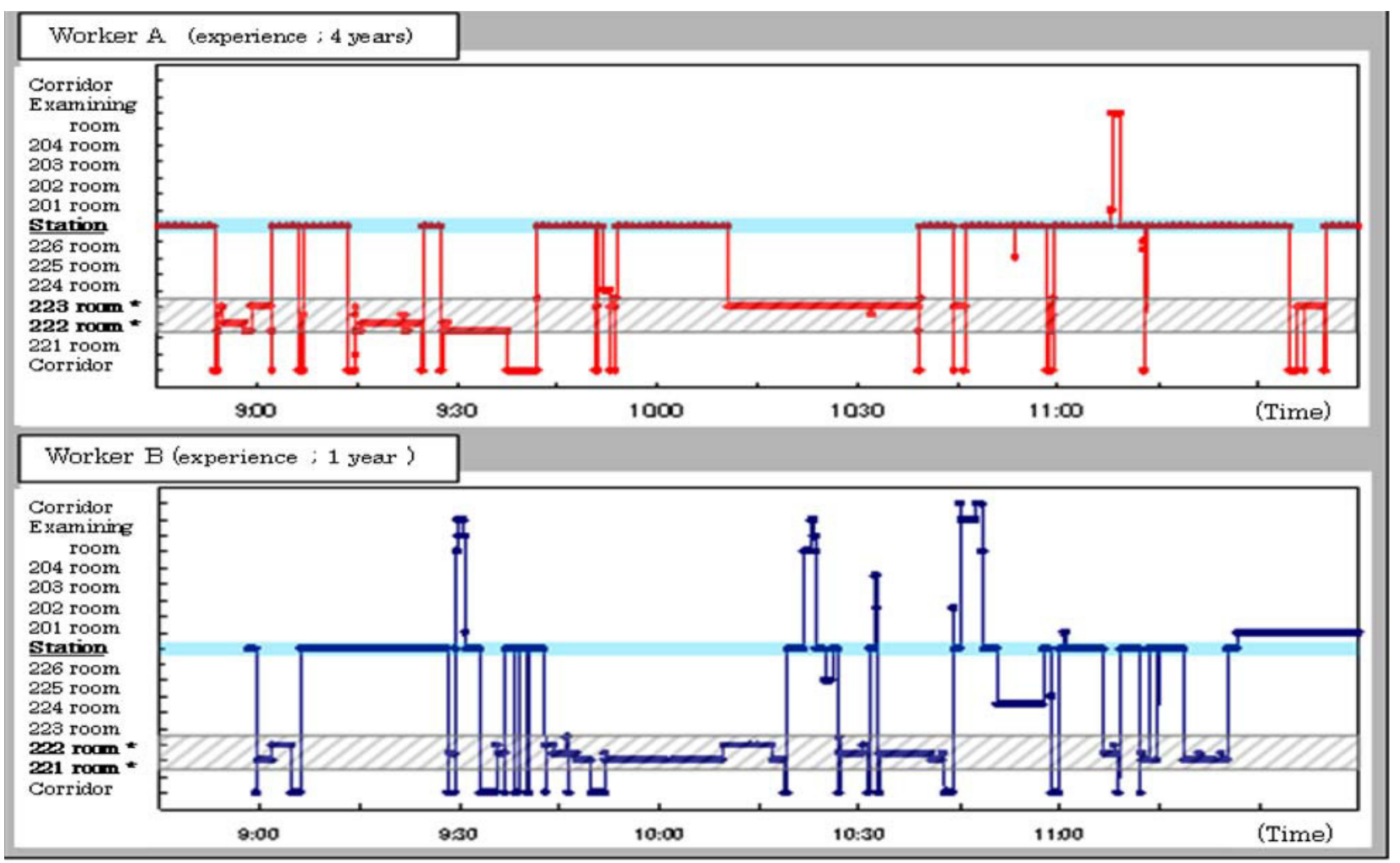

Fig.1 Comparison of moving route in workflow line surveying of hospital (*; the room in attendance) 